\title{
LA INEQUIDAD EN SALUD AFECTA EL DESARROLLO EN EL PERÚ
}

\author{
INEQUITY IN HEALTH AFFECTS THE DEVELOPMENT IN PERU
}

Francisco Sánchez-Moreno $0^{1, a, b}$

Academia Peruana de Salud. Lima, Perú

a Doctor en Medicina; ${ }^{b}$ Presidente emérito de la Academia Peruana de Salud; ex decano de la Facultad de Medicina de la Universidad Nacional Mayor de San Marcos.

Recibido: 03-09-2013; Aprobado: 06-11-13

\begin{abstract}
RESUMEN
Las diferencias injustas y evitables en el ejercicio del derecho a la salud coexisten con la Declaración Universal de Derechos Humanos desde 1948. Algunas causas de la persistente inequidad se infieren después de Alma-Ata, tales como financiación insuficiente, desarrollo de los sistemas nacionales de salud sin adecuada priorización, y desde la década de 1980 concertación económica para introducir el modelo de mercado en salud. En la actualidad en Perú, la salud sigue siendo un ámbito de escasos avances, ostensible inequidad y participación limitada en el desarrollo. Continúan las insuficientes políticas del siglo XX y es recurrente la pérdida de oportunidades, incluyendo la que brinda el incremento del valor de las exportaciones desde hace más de una década. Para una reforma de salud exitosa hacia la equidad y el desarrollo, se requiere concertar una política de Estado, establecer financiación moderna y equitativa del aseguramiento social y terminar con los vaivenes del sistema nacional de salud.
\end{abstract}

Palabras clave: Derecho a la salud; Equidad en salud; Sistemas nacionales de salud; Seguridad social; Atención primaria de salud; Gastos en salud; Perú (fuente: DeCS BIREME).

\begin{abstract}
Unfair and avoidable differences in exercising the right to health coexist with the Universal Declaration of Human Rights since 1948. Some causes of the persistent inequality inferred after Alma-Ata are insufficient funding, development of national health systems without adequate prioritization, and since the $80 \mathrm{~s}$ with economic consensus to introduce the health market model. Health in Peru is still an area of little progress, ostensible inequality and limited participation in development. Policies in the 20th century are still insufficient; and missing opportunities like increasing the value of exports overa decade ago is a recurring issue. For a health reform to be successful in terms of equality and development, it is necessary to agree on a state policy, establish a modern and equitable social security funding system and eradicate the inconsistencies in the national health system.
\end{abstract}

Key words: Right to health; Equity in health; National health systems; Social security; Primary health care; Health expenditures; Peru (source: MeSH NLM).

\section{INTRODUCCIÓN}

Por inequidad en salud entendemos las diferencias injustas y evitables en el ejercicio de los derechos fundamentales a la salud y la seguridad social en salud $y$, principalmente, en el acceso a los servicios y prestaciones de salud. Estas disparidades resultan de las condiciones en que la población nace, crece, vive y envejece en relación con los factores o determinantes de la salud, entre ellos, el desempeño del sistema nacional de salud y su financiamiento ${ }^{(1-4)}$.

Se trata de diferencias no naturales que afectan la vida y los riesgos de enfermar y morir, interfieren el desarrollo humano y social con múltiples efectos -inclusive en el crecimiento económico- y que, asimismo, lindan con un componente ético implícito en la Declaración de AlmaAta, cuando precisa que la grave desigualdad existente en el estado de salud de la población "...es política, social y económicamente inaceptable..." (acápite II) ${ }^{(5)}$. Es posible, en consecuencia, valorar el desarrollo de un país por el estado de salud de su población, la distribución de los problemas de salud en el espectro social y el grado de protección de las personas con relación a los derechos humanos fundamentales ${ }^{(6-10)}$.

\section{INEQUIDAD MUNDIAL EN SALUD}

La Declaración Universal de Derechos Humanos aprobada y proclamada por la Asamblea General de las Naciones Unidas el 10 de diciembre de 1948- determinó

Citar como: Sánchez-Moreno F. La inequidad en salud afecta el desarrollo en el Perú. Rev Peru Med Exp Salud Publica. 2013;30(4):676-82. 
el derecho a la salud y al bienestar (artículo 25) y el derecho a la seguridad social (artículo 22) (11):

\section{“Artículo 25}

1. Toda persona tiene derecho a un nivel de vida adecuado que le asegure, así como a su familia, la salud y el bienestar, y en especial la alimentación, el vestido, la vivienda, la asistencia médica y los servicios sociales necesarios; tiene, asimismo, derecho a los seguros en caso de desempleo, enfermedad, invalidez, viudez, vejez u otros casos de pérdida de sus medios de subsistencia por circunstancias independientes de su voluntad.

2. La maternidad y la infancia tienen derecho a cuidados y asistencia especiales. Todos los niños, nacidos de matrimonio o fuera de matrimonio, tienen derecho a igual protección social."

\section{“Artículo 22}

Toda persona, como miembro de la sociedad, tiene derecho a la seguridad social, y a obtener, mediante el esfuerzo nacional y la cooperación internacional, habida cuenta de la organización y los recursos de cada Estado, la satisfacción de los derechos económicos, sociales y culturales, indispensables a su dignidad y al libre desarrollo de su personalidad."

El Perú suscribió la Declaración en 1948 y le dio inmediato vigor normativo. La Constitución Política, en sus artículos 55 y 56 inciso 1 y la cuarta disposición final, establece //...que las normas relativas a los derechos que la Constitución reconoce se interpretan de conformidad con la Declaración Universal de Derechos Humanos y con los tratados y acuerdos internacionales ratificados por el Perú...// (12).

Sin embargo, los avances han sido extremadamente disímiles en las distintas naciones y los derechos humanos coexisten con inequidad en salud por más de seis décadas.

\section{ALMA-ATA 35 AÑOS DESPUÉS}

Al término de la Conferencia Internacional más importante habida en salud, la Declaración de Alma-Ata definió el 12 de septiembre de 1978 que: "La atención primaria de salud es la asistencia sanitaria esencial basada en métodos y tecnologías prácticos...// ... puesta al alcance de todos los individuos y familias de la comunidad mediante su plena participación y a un costo que la comunidad y el país puedan soportar, en todas y cada una de las etapas de su desarrollo...// La atención primaria forma parte integrante tanto del sistema nacional de salud, del que constituye la función central y el núcleo principal como del desarrollo social y económico global de la comunidad....// Representa el primer nivel de contacto de los individuos, la familia y la comunidad con el sistema nacional de salud, llevando lo más cerca posible la atención de salud al lugar donde residen y trabajan las personas, y constituye el primer elemento de un proceso permanente de asistencia sanitaria.// ...Es posible alcanzar un nivel aceptable de salud para toda la humanidad en el año 2000 mediante una utilización mejor y más completa de los recursos mundiales...// en particular para acelerar el desarrollo social y económico..." (5).

Después de 35 años pueden inferirse tres causas elementales en la frustración de la meta "salud para todos": desarrollo de los sistemas nacionales de salud sin adecuada prioridad; insuficiente financiación de la atención primaria complementada con prestaciones integrales en los otros niveles; y concertación internacional de contenido económico para establecer desde los años ochenta el modelo neoliberal de mercado en salud. Un cambio resulta poco probable mientras persistan ${ }^{(2,13,14)}$.

La Declaración de Alma-Ata especifica que la atención primaria "...forma parte integrante del sistema nacional de salud, del que constituye la función central y el núcleo principal..." (5). Sin embargo, no existieron acciones internacionales continuas para promover el desarrollo de los sistemas nacionales de salud y alcanzar desempeños eficientes en sus cuatro funciones principales: rectoría, financiamiento incluyendo recaudación, prestación de servicios y generación de recursos humanos y físicos ${ }^{(13)}$. Años después se pudo comprobar que los países con resultados satisfactorios en atención primaria y cuidado integral de la salud fueron mayoritariamente los que desarrollaron su sistema nacional de salud, como los casos de Francia e Italia en Europa con el modelo de seguridad social y sus principios de solidaridad y universalidad entre otros (13-18). En América Latina, salvo Costa Rica (y Cuba con el modelo de integración estatal), no hubo énfasis para desarrollar los sistemas nacionales de salud y por ende sus responsabilidades centrales de atención primaria y financiamiento $(1,2,13,14)$.

En lo que atañe a la financiación de la asistencia sanitaria, el enunciado de Alma-Ata "...a un costo que la comunidad y el país puedan soportar..." corresponde a una traducción al español de "...at a cost that community and country can afford...", concepto éste más cercano a "solventar", como recientemente ha sido mencionado ${ }^{(5,19)}$. Resulta evidente que después de 
la Declaración, no se contó con acciones continuadas a nivel mundial para promover procesos nacionales orientados a lograr recursos económicos suficientes y solventar los costos, por ejemplo mediante la extensión de la seguridad social. En consecuencia, la mayoría de países careció de factibilidad para el acceso de la gran población a la atención primaria complementada con prestaciones integrales en los otros niveles ${ }^{(2,13,14)}$.

La tercera causa -universalmente conocida- fue desde los años ochenta del siglo $X X$ y sigue siendo en la mayoría de países, una controvertida acción de contenido económico contraria a la equidad, la solidaridad, a la atención primaria, la rectoría del Estado en los sistemas nacionales de salud y a la seguridad social y su financiamiento. Consistió en el masivo impulso de la banca internacional para establecer el modelo neoliberal de mercado en salud. Ciertamente, en el "mejor de los casos" ganó el mercado y perdió la humanidad, pues esta acción resultó muy negativa para la salud y el desarrollo humano y social en muchísimos países, y dramática en América Latina. ¿Es acaso imposible construir humanidad y economía con razonable equilibrio? ${ }^{(1,2,13,14,20)}$.

\section{PERÚ: VAIVENES DEL SISTEMA DE SALUD E INEQUIDAD}

Cuando a fines de los años sesenta y comienzos de los setenta del siglo XX el Perú perdió la democracia participativa con un abrupto cambio hacia el estatismo, perdió también el ritmo que la mayoría de países de América del Sur siguió en salud y seguridad social, y desde hace más de 40 años quedó en los últimos lugares. Con un nuevo giro de gobierno, la política estatista se desvaneció a partir de agosto de 1975 hasta desaparecer. Pero en salud prosiguió una larga inestabilidad ${ }^{(13,21)}$.

Coincidiendo con la época de la Declaración de Alma-Ata, entre 1978 y 1979, nos correspondió participar en la alta dirección del Ministerio de Salud en un avance pionero, como fue la creación del Sistema Nacional de Servicios de Salud (SNSS) por Decreto Ley 22365. Esto ocurrió antes de las reformas de los sistemas nacionales de salud en Chile (1980), Brasil (1990), Colombia (1993) y Ecuador (2008). El sistema tuvo año y medio de fructífera y organizada coordinación de todas las instituciones de salud a través del Consejo Nacional de Salud y los consejos regionales, y concretó logros trascendentes para construir equidad, como el primer plan nacional de atención primaria de salud, un importante programa nacional de medicamentos básicos y la propuesta y desarrollo del Régimen de Prestaciones de Salud del Seguro Social para la extensión del seguro social a la familia mediante Decreto Ley 22482. El innovador proceso fue singularmente destacado por el director general de la Organización Mundial de la Salud doctor Halfdan T. Mahler con la visita específica que hizo al Perú en $1980^{(13,14,21)}$.

Por falta de una política de Estado, este alentador inicio tuvo un devenir increíble: como en ningún otro país, el sistema nacional de salud afronta desde entonces vaivenes permanentes. Sin previa evaluación, fue desacelerado en 1980, desactivado en 1985 y derogado en 1990. Por Decreto Legislativo 584 fue creado el Sistema Nacional de Salud (SNS) en 1990, nunca fue reglamentado, se crearon los sistemas locales de salud (Zonadis) en 1992, fueron desactivados en 1994 y eliminados el 2002. Por último, el actual Sistema Nacional Coordinado y Descentralizado de Salud (SNCDS) fue creado por Ley 27813 el año 2002, desacelerado el 2003, nunca tuvo prioridad, se intentó derogarlo el 2008, se pretendió desnaturalizarlo con varios proyectos de Ley el 2012 y ha entrado a un proceso de reforma en el presente año con facultades legislativas otorgadas al Poder Ejecutivo por el Congreso de la República ${ }^{(13,14,21,22)}$.

Es evidente que la discontinuidad es la característica principal del sistema peruano desde su creación hace 35 años, con el resultante desempeño ineficiente y sin evaluación, lo que explica la gran inequidad y el atraso con relación a los sistemas de salud de América del Sur. Si cada gobierno continuara estableciendo "su" política de salud no podrá haber equidad ni desarrollo. La concertación de una moderna política de Estado en salud es indispensable ${ }^{(13,14,23)}$.

Como consecuencia de la desorganización del sistema y su deficiente financiamiento, tampoco hay equidad en el campo de los recursos humanos: bajísimas remuneraciones, conflictividad permanente, múltiples deficiencias en la formación y gestión, insuficiencia ostensible de profesionales en hospitales y en todos los niveles de atención, y altísima y creciente migración al extranjero jamás ocurrida, como lo demuestran las migraciones sin retorno. De los médicos: 834 el 2011, 1739 el 2012 y 2532 de enero a junio del 2013; y de los enfermeros: 1349 el 2011, 2243 el 2012 y 2357 de enero a junio del $2013^{(2,14,24)}$.

\section{EXPRESIONES DE INEQUIDAD EN SALUD}

Dos tercios de la población peruana continúan excluidos de los derechos a la salud y la seguridad social, así como del acceso a los servicios de atención integral. 
Para beneficio de muy pocos y como sucedió en diversos países, el apoyo de las instituciones financieras internacionales al modelo de mercado desplazó en la decisión política a la seguridad social, con lo cual esta perdió prioridad desde los dos últimos decenios del siglo XX. Hoy el aseguramiento social en salud, su financiación, el acceso a los servicios y la calidad son más inequitativos y excluyentes ${ }^{(25-29)}$.

La inequidad es extrema en las enfermedades de alto costo y existen, asimismo, otros casos lacerantes. Por ejemplo, en gran parte del país es imperceptible el cuidado de la salud mental. Existen más de 66 mil personas con $\mathrm{VIH}$-adquirido $97 \%$ por vía sexual- de las que apenas la mitad conoce que lo padece, no obstante son transmisores cotidianos de la infección. $Y$ han recrudecido antiguos problemas como el retroceso en el control de la tuberculosis con mayor resistencia a los medicamentos; o las desesperantes e innumerables frustraciones en los cada vez más congestionados servicios de emergencia, consulta, cirugía y hospitalización; o las cotidianas insuficiencias de atención, medicamentos y equipos en los distintos niveles regionales, provinciales y distritales ${ }^{(2,25,26)}$.

Hay, además, inequidades motivadas por injusticias de índole diversa, como los elevados riesgos de embarazos tempranos y no deseados en adolescentes, o por consumo de drogas. Otra que no puede dejar de mencionarse, de repercusión internacional porque atenta severamente contra el derecho a la salud física y mental de las mujeres; es el caso de la anomalía del embarazo con feto anencefálico, en el que increíblemente se obliga a la gestante a continuarlo hasta el final ${ }^{\left({ }^{30}\right)}$, no obstante el concluyente mandato de la Ley 28189 que dice: "...el diagnóstico y certificación de la muerte de las funciones encefálicas equivale a la muerte de la persona para efectos de proceder a los trasplantes de órganos...". Sin duda alguna, la ausencia de funciones ocurre igualmente por inexistencia del encéfalo. Pero hasta el presente no se ha concretado la solución (31).

\section{FINANCIAMIENTO INSUFICIENTE CON DISEÑO DE 1935}

La Ley de Aseguramiento 29344 de abril del 2009 no afrontó la insuficiencia de sustento financiero a fin de lograr una disminución efectiva de la inequidad. Apenas consideró incrementos presupuestales inestables y, en el contexto neoliberal con seguro privado alternativo en que fue elaborada, hubo omisión en diseñar el financiamiento contributivo factible de los trabajadores informales. El Fondo Intangible Solidario de Salud (Fissal) nada significativo resuelve y la reciente reorganización de Seguro Social de Salud (Essalud) tampoco ha tenido ni tendrá repercusión alguna ${ }^{(14,25,32,33)}$.

Con el diseño original de 1935, la financiación del aseguramiento y la recaudación subsisten insuficientes. Si bien los logros del precursor de la seguridad social peruana Edgardo Rebagliati fueron sobresalientes hace 78 años, entonces la población peruana era poco más de seis millones de habitantes. Pero ahora es más de 30 millones y los hospitales y emergencias resultan exiguos y totalmente congestionados ${ }^{(14,27)}$.

El diseño de los aportes debió ser modernizado hace dos o tres décadas, pues con inequidad financiera extrema excluye a la creciente y ya enorme población ocupada con trabajo informal. Tal exclusión afecta severamente el financiamiento del Seguro Integral de Salud y el Seguro Social de Salud, porque del total de trabajadores peruanos - muchos teniendo ingresos de nivel medio y alto- solo el $25,1 \%$ cotiza al seguro social, cuando en América del Sur aporta el $46 \%$, en Argentina el $70 \%$ y en varios países de Europa cerca del $100 \%{ }^{(14,34)}$.

Como resultado, en la financiación del aseguramiento la inequidad es contundente. El año 2012 el Seguro Integral de Salud (SIS) tuvo apenas un gasto de 35 dólares per cápita al año (para 42,1\% de la población), mientras en Essalud fue 196 dólares (30,4\% de la población) y en las entidades prestadoras de salud (EPS) 542 dólares (apenas 1,7\% de la población), según sus propias cifras institucionales. Más aun, el $20 \%$ de los peruanos carece de aseguramiento en salud ${ }^{(35-38)}$.

En América del Sur el gasto per cápita en salud fue el 2009 de 493 en promedio y en los países de Europa entre 3000 y 7000 dólares al año, de acuerdo al último informe de la Organización Mundial de la Salud publicado el $2012{ }^{(39)}$. Esta severa inequidad debe ser la primera prioridad en cualquier reforma de salud en el Perú y es elemental que se informe anualmente sobre las cifras per cápita del gasto en salud de todas las instituciones nacionales ${ }^{(14)}$.

\section{IMPRESCINDIBLE: FINANCIAMIENTO MODERNO Y EFECTIVO}

La seguridad social universal es factible, pero en un largo plazo no menor de 15 años. Su extensión a todos requerirá un gasto nacional anual per cápita en salud estimado en más de 700 dólares constantes. Esto implicará gradualidad en el cumplimiento pleno de los principios de la seguridad social debido a la complejidad del proceso de financiamiento. 
Hace once años, en enero del 2002, en el Ministerio de Salud se elaboró con la consultoría de expertos de la seguridad social francesa, una propuesta moderna de financiamiento y recaudación del aseguramiento universal, a fin de incorporar progresivamente a toda la población peruana. En febrero de 2004 se preparó el respectivo Proyecto de Ley, pero por motivos políticos ocasionales todo fue discontinuado ${ }^{(14,17,25)}$.

En el Perú la financiación moderna y equitativa del aseguramiento es posible, y para una reforma de salud exitosa, imprescindible. Al respecto, en el año 2011 la Academia Peruana de Salud presentó a los poderes públicos un Proyecto de Ley de Aseguramiento Universal Solidario en Salud. La clave del cambio es el financiamiento mixto, porque el financiamiento solamente subsidiado o impositivo es insuficiente y en las naciones de menor desarrollo tiene limitaciones insalvables.

Debe quedar muy claro, entonces, que el Aseguramiento Social Universal en Salud está al alcance del país si es diseñado con financiamiento mixto. Por tanto, resulta imprescindible normar el aporte contributivo solidario y obligatorio de los trabajadores formales, informales e independientes, en proporción a sus ingresos. Como es en la empresa con el trabajador formal y con base en la equidad financiera, se propendería a que el aporte del trabajador informal sea parte del valor del producto que vende (ropa, bebidas, etc.) o del servicio que presta (taxi, vigilancia, mensajería, etc.). Los pagos se harían con tecnología moderna y para facilitar y garantizar la recaudación, quien no tiene registro único de contribuyente (RUC) utilizaría el documento nacional de identidad (DNI) al comprar o vender por un monto mínimo a determinar. Con apoyo del Registro Nacional de Identificación y Estado Civil (Reniec) habría un Registro Nacional de Aseguramiento ${ }^{(14,17,18,25,32,33)}$.

Si el aporte contributivo del asegurado fuera igual o mayor al monto mínimo que se determine actuarialmente, se adscribiría a Essalud; y -si fuera menor- al SIS. Esto motivaría permanentemente a los asegurados a incrementar sus aportes para pertenecer a Essalud. El volumen de población que superaría el monto mínimo del aporte contributivo sería elevado y progresivo. Se generaría así en Essalud un aumento gradual de asegurados, con las positivas consecuencias de un mayor financiamiento, un desarrollo sin precedentes del aseguramiento y el incremento de la participación privada complementaria (no alternativa) al seguro social ${ }^{(14,25,27,32,33)}$.

\section{POR UNA MAYOR PRESENCIA DE LA SALUD EN EL DESARROLLO}

Con escasos avances, la salud continúa siendo un ámbito de limitada participación en el desarrollo del Perú, el cual requiere un conjunto de acciones y efectos vinculados al desarrollo humano y social, al crecimiento económico, la producción, las inversiones, el comercio internacional y el buen manejo público. Cuando no se otorga la importancia que corresponde al ser humano, a su salud, su educación y a los determinantes y elementos relacionados, el desarrollo es afectado, como puede evidenciarse cotidianamente en el caso peruano en todos los ámbitos sociales ${ }^{(1,2)}$.

La salud es resultante de procesos políticos, económicos y sociales, que impactan de distintas maneras. El derecho a la salud, el acceso a los servicios, el aseguramiento social, el sistema nacional de salud y la situación del sector en general no muestran avances suficientes, debido principalmente a una rectoría que involuciona permanentemente con la política de mercado y determina la ausencia de decisiones y estrategias modernas, como por ejemplo para un financiamiento equitativo, hoy al alcance del país ${ }^{(1,2,32,33)}$.

Si bien la esperanza de vida y otros indicadores han continuado su tendencia a mejorar en el Perú - como en la gran mayoría de naciones en razón del desarrollo mundial- subsiste un retraso importante en salud que ha llevado al país desde el nivel medio de América del Sur en los años 50 del siglo XX, a ocupar los últimos lugares en la primera década del siglo XXI.

La salud carece de prioridad y sobreviven aún las insuficientes políticas del siglo XX. Lo usual es la pérdida de oportunidades, inclusive la que sigue brindando al Perú la nueva realidad económica mundial desde hace más de una década por el incremento del valor de las exportaciones. Aprovecharla debidamente es un enorme reto para el desarrollo nacional $(1,2,14,25)$.

\section{REFLEXIONES FINALES: HACIA UNA POLÍTICA DE ESTADO EN SALUD}

Emprender la transformación por el derecho fundamental a la salud, la equidad y el desarrollo requiere decisión, otorgar la prioridad que corresponde a la salud y concertar con todos el logro de una política de Estado ${ }^{(1,2,14,21,25,32)}$.

Lo más urgente es establecer el aseguramiento social financiado con estrategias efectivas, recaudación moderna e inversión nacional mayor al promedio de América del Sur. Con este objeto urge concertar 
una ley de aseguramiento social universal en salud: obligatorio, factible, efectivo, progresivo y con la moderna estrategia de incorporación contributiva de los trabajadores informales, a fin de recaudar con equidad sus aportes a través del valor de las ventas, servicios o ingresos, como ocurre en el Perú desde 1936 con los trabajadores formales; y contar con la importante participación complementaria del sector privado; ciertamente sin seguros alternativos al seguro social, porque estos incrementan la inequidad y la exclusión.

La prioridad siguiente es terminar con los vaivenes del sistema nacional de salud, desarrollar sus funciones, alcanzar pronto el desempeño eficiente, evaluarlo periódicamente y fortalecer la débil rectoría sectorial. El modelo coordinado de sistema propende a una transición progresiva hacia la seguridad social universal y esta ha demostrado en distintos países ser el modelo de sistema de salud más completo y equitativo. Debe iniciarse el proceso de manera que la atención en el primer y segundo nivel del sistema responda a los requerimientos de la población, con medicamentos, infraestructura, equipos y recursos humanos capacitados y debidamente remunerados. Es necesario que las universidades realicen docencia de pre y posgrado de calidad en las materias de sistema nacional de salud y aseguramiento social universal, como en los países que han desarrollado en salud.

Finalmente, con la reciente promulgación de la Ley 30023 surge en el Perú la nueva gran oportunidad de concertar un proceso progresivo de participación de todos los peruanos con motivo del "Día nacional de la salud y del buen trato al paciente", como símbolo del impulso que es imprescindible dar a la salud para mejorar el cuidado integral y disminuir la inequidad, promoviendo el derecho a la salud, el aseguramiento social y el desarrollo nacional, regional y local (40).

Descargo de responsabilidad: las opiniones y afirmaciones contenidas aquí son propias del autor.

Fuentes de financiamiento: autofinanciado.

Conflictos de interés: el autor declara no tener conflictos de interés.

\section{REFERENCIAS BIBLIOGRÁFICAS}

1. Academia Peruana de Salud. Historia de la Salud en el Perú. Salud y desarrollo. Lima: Academia Peruana de Salud; 2009.

2. Academia Peruana de Salud. Historia de la Salud en el Perú 2007-2011. Salud y desarrollo. Lima: Academia Peruana de Salud; 2013.

3. Caballero JF. La teoría de la justicia de John Rawls [Internet]. Iberoforum. 2006; II(I):1-22 [citado el 10 de agosto del 2013]. Disponible en: http://www.uia.mx/actividades/ publicaciones/iberoforum/2/pdf/ francisco_caballero.pdfhttp://www. uia.mx/actividades/publicaciones/ iberoforum $/ 2 / \mathrm{pdf} /$ francisco_ caballero.pdf

4. Whitehead M. The concepts and principles of equity and health [Internet]. Geneva: World Health Organization; 2000 [citado el 14 de agosto del 2013]. Disponible en: http://salud.ciee.flacso.org.ar/flacso/ optativas/equity_and_health.pdf

5. Organización Mundial de la Salud. Informe de la Conferencia Internacional sobre Atención Primaria de Salud. Alma-Ata, URSS, 6-12 de septiembre de 1978. Ginebra: OMS; 1978.

6. Organización Panamericana de la Salud. Salud: una condición para el desarrollo. En: Pro salute novi mundi. Historia de la OPS. Washington, DC: OPS; 1992. p. 86-115; 268-77.

7. Organización Panamericana de la Salud. Macrodeterminantes de la salud en el desarrollo humano sostenible. En: La salud en las Américas. Washington, DC: OPS; 2002. p. 91-136.

8. Seclén S. Pobreza e inequidad en salud. En: Coyuntura. Análisis económico y social de actualidad. Lima: Pontificia Universidad Católica del Perú; 2008.

9. Miranda C. La pobreza en el Perú y el mundo. Rev Cpo Med Hosp Almenara. 2013;27(1):71-9.

10. Organización Mundial de la Salud. Subsanar las desigualdades en una generación: alcanzar la equidad sanitaria actuando sobre los determinantes sociales de la salud. Ginebra: OMS; 2009.

11. Declaración Universal de Derechos Humanos. Asamblea General de las Naciones Unidas, 10 de diciembre de 1948.
12. Perú. Constitución Política. 29 de diciembre de 1993.

13. Academia Peruana de Salud. Historia de la Salud en el Perú. Sistema nacional de salud. Lima: Academia Peruana de Salud; 2008.

14. Academia Peruana de Salud. L Foro "Salud y Desarrollo". El futuro de la salud en el Perú. Rev Acad Peru Salud. 2013;20(1):30-60.

15. Organización Mundial de la Salud. Mejorar el desempeño de los sistemas de salud. Informe sobre la salud en el mundo. Ginebra: OMS; 2000.

16. Centro Interdisciplinario de Estudios sobre el Desarrollo Latinoamericano. La seguridad social en América Latina; seis experiencias diferentes. Colombia, Costa Rica, Cuba, Paraguay, Perú y Venezuela. Buenos Aires: Konrad Adenauer Stiftung A.C. Ciedla; 1996.

17. Perú, Ministerio de Salud. Informe final de la Comisión de Alto Nivel para reiniciar la organización del Sistema Nacional de Salud R.M. No 463-2001-SA/DM. Lima: MINSA; 2002.

18. World Health Organization. Health systems: finding new strength. En: The 
world health report, cap. 4. Geneva: WHO; 2004.

19. Solari L. Atención primaria de salud: un compromiso pendiente [editorial]. Rev Peru Med Exp Salud Publica. 2013;30(2):173-4.

20. De Vos P; Van Der Stuyft P. Determinantes sociopolíticos de las políticas internacionales de salud. Rev Peru Med Exp Salud Publica. 2013;30(2):288-96.

21. Academia Peruana de Salud. Historia de la Salud en el Perú 2007-2011. Sistema nacional de salud. Lima: Academia Peruana de Salud; 2013.

22. Perú, Ministerio de Salud; Consejo Nacional de Salud. Of. № 255-2012-SECCOR/CNS, 21.02.2012 y exposición documentada del Secretario de Coordinación del Consejo Nacional de Salud en la Comisión de Salud y Población, grupo de trabajo sobre reforma del sistema nacional de salud y seguridad social universal. Congreso de la República, Lima, Perú, 31.01.2012.

23. Organización Panamericana de la Salud. La salud pública en las Américas. Nuevos conceptos, análisis del desempeño y bases para la acción. Washington, DC: OPS; 2002. Publicación científica y técnica 589.

24. Perú, Ministerio del Interior, Superintendencia Nacional de Migraciones. Número de profesionales médicos, enfermeras, obstetrices y odontólogos migrantes sin retorno 20042013. Of. $\mathrm{N}^{\circ}$ 198-2013-MigracionesTICE; 23.07.2013. Lima: Ministerio del Interior; 2013.

25. Academia Peruana de Salud. Historia de la Salud en el Perú 2007-2011. Aseguramiento social en salud. Lima: Academia Peruana de Salud; 2013.
26. Sánchez-Moreno F. Aseguramiento universal: Los procesos en el Perú y EE.UU. Sesión conjunta de la Academia Nacional de Medicina y la Academia Peruana de Salud. Rev Acad Peru Salud. 2010;17(2):26-34.

27. Sánchez-Moreno F. Reforma del sistema nacional de salud y seguridad social universal. Exposición en la Comisión de Salud y Población del Congreso de la República. Rev Acad Peru Salud. 2011;18(2):4-14.

28. Organización Panamericana de la Salud. Contribuyendo al financiamiento sostenible de sistemas de salud de cobertura universal. Lima: Editor Ramón Granados; 2011.

29. Consorcio de Investigación Económica y Social. Observatorio de la Salud. Promoviendo el derecho a la salud de los más pobres: cinco estudios de interés. Lima: Ediciones Nova Print SAC; 2012.

30. Comité de América Latina y el Caribe para la Defensa de los Derechos de la Mujer, CLADEM. Caso KL vs. Perú, comunicación 1153. Bol Programa Litigio Internacional. 2003;1(5):3.

31. Perú, Ley No 28189, Ley General de Donación y Trasplante de Órganos y/o Tejidos Humanos, 2004; y Reglamento, Decreto Supremo $\mathrm{N}^{\circ}$ 014-2005-SA.

32. Academia Peruana de Salud. Proyecto de Ley de Aseguramiento Universal Solidario en Salud y XIV Coloquio Académico. Rev Acad Peru Salud. 2010;17(2):5-18.

33. Academia Peruana de Salud. XV Coloquio Académico. Período de gobierno 2011-2016 en salud. Análisis y propuestas. Rev Acad Peru Salud. 2011;18(1):11-8.
34. Casali P. La iniciativa del piso de protección social. Contribuyendo al financiamiento sostenible de sistemas de salud de cobertura universal (OIT). I Conferencia Internacional de Aseguramiento Universal en Salud. Lima, Perú nov. 2010. Lima: OPS; 2011.

35. Perú, Congreso de la República. Comisión de Salud y Población, documentos solicitados y recibidos por el grupo de trabajo sobre reforma del sistema nacional de salud y seguridad social universal. Lima; feb. 2012.

36. Perú, Ministerio de Salud. Seguro Integral de Salud. R. Jefatural No 006 2012/SIS, 06.01.2012.

37. Perú, Seguro Social de Salud. Essalud, Gasto anual per cápita en salud ejecutado el 2012. Of. $\mathrm{N}^{\circ}$ 366-SGESSALUD-2013; 3.set.2013

38. Perú, Ministerio de Salud. Superintendencia Nacional de Aseguramiento en Salud. Gasto anual en prestaciones de salud en el Sistema Entidades Prestadoras de Salud 2012. Of. No 002-2013-Sunasa/ACCINF;7. ago.2013.

39. Organización Mundial de la Salud. Estadísticas sanitarias mundiales; 2009. Ginebra: OMS; 2009.

40. Academia Peruana de Salud. Historia de la Salud en el Perú 2007-2011. Hacia el "Día Nacional de la Salud". Lima: Academia Peruana de Salud; 2013.

Correspondencia: Francisco Sánchez-Moreno Dirección: Calle Manuel Segura $N^{\circ} 122$ - Of. 704, Lima 14, Perú

Teléfono (511) 470-3798

Correo electrónico: samorel@speedy.com.pe 